\title{
Therapeutic application of Botulinum Toxin A in patients with local muscle dystonia and oral dyskinesia
}

\author{
O. Orlova ${ }^{1}$, M. I. Soikher ${ }^{2}$, M. G. Soikher ${ }^{2}$, L. Mingazoval ${ }^{1}$, V. Kotlyrov ${ }^{1}$, G. Slavicek ${ }^{3}$ \\ ${ }^{1}$ Medical Institute Sechenov, Moscow Medical Academy, Moscow, Russia \\ ${ }^{2}$ Institute of Biotechnologies and Interdisciplinary Dentistry, Moscow, Russia \\ ${ }^{3}$ Steinbeis Transfer Institut Biotechnology in Interdisciplinary Dentistry, Steinbeis Hochschule Berlin, Berlin, Germany
}

Received November 25, 2009; Accepted January 30, 2010

Botulinum Toxin Type A has been introduced as new therapeutic regime in neurological, cosmetic, urinary, gastrointestinal and pain-related conditions. The use of Botulinum Toxin A in craniomandibular disorders is discussed controversially.

Local muscle dystonia is defined as movement disorder in combination with sustained muscle contractions. Contortion, repetitive movements and atypical posture are frequently seen symptoms in such cases. The movements are involuntary and often painful. A single muscle or a group of muscles, even the whole body may be involved. Oral dyskinesias are involuntary repetitive movements of the mouth and face. Severe bruxism can be included in this form of movement disorder.

Within this study, patients diagnosed with severe bruxism or local muscle dystonia including oral dyskinesia were examined with electromyography before and after treatment. Subjects were randomly allocated to either myo-relaxation medication and relaxation splint therapy or to Botulinum Toxin A injection therapy. Surface EMG parameters of chewing and neck muscles were used to judge the treatment effects. A positive impact of BTA on EMG-characteristics, reflecting the functional condition of facial muscles, had been detected. BTA injections seem to have a positive effect in patients with bruxism and local muscle dystonia. An increased quantity of mandibular movements and a better coordination can be observed after BTA injection. In comparison with a standard myo relaxation therapy, the effects of BTA treatment are more distinct and long standing.

BTA appears to be a promising therapeutic regime in patients with focal muscle dystonia and oral dyskinesia. A confirmed diagnosis and lege artis application by experienced clinicians constitute as sine qua non. Further studies are required to improve our knowledge in Botulinum Toxin A treatment of muscle dystonia and dyskinesia.

Keywords: muscle hypertonia, botulinum toxin type A, parafunction, abnormal abrasion, myofascial pain syndrome, bruxism

Correspondence: Marina Soikher, Institute of Biotechnologies and Interdisciplinary Dentistry, 123123 Moscow; Konsomolsky Prospect 32/2, Russia. E-mail: marina-soiher@yandex.ru

\section{Introduction}

Growing interest on muscle pathology becomes noticeable, as muscle pain often plays a major role in clinical records $[7,8]$. Epidemiological studies proved that approximately $5 \%$ people worldwide suffer rather severe pain in masticatory muscles to an extent, which requires treatment [41]. Treatment costs of muscle dysfunction syndrome in combination with temporomandibular joint dysfunction are calculated to be similar to costs of the therapy for malignant tumours. Dysfunction of head and neck muscle are frequently diagnosed and can be found on the 3rd place after caries and periodontal diseases [4].

Myalgia contributes up to $97 \%$ all cases of chronic orofacial pain, although muscle pain is clinically often related to teeth (odontoalgia). Inadequate and inefficient treatment is the logical consequence [61]. Diagnosis and revealing causal relationship are difficult due to variety of clinical symptoms in facial myalgia. Moreover, pathological processes in the area of the face, even slightly expressed, often become of high priority for patients and they may develop neurotic reactions and aggravation of the pain $[10,11]$.

Masticatory muscle diseases are multi-factorial. The progress of such a condition is accelerated by combination of particular factors: changes in form of muscle apparatus, occlusal abnormalities, pathologies in posture (cervical thoracic spine flexion, asymmetry of shoulders, scapulas, shortening of a leg etc. [7, 8, 19, 20, 42, 61]). Myalgia may influence central nervous system. Abnormal muscle function is due to changes in relaxation process of the muscular system, resulting in muscle hypertonus. Painful muscle areas of various density and size are formed. Excessive and continuous activity of masticatory muscles causes hypertrophy in combination with an increase of muscle power and muscle mass.

Hypertonic masticatory muscles are often triggered by occlusion $[12,21,50]$. Abnormality in occlusion leads to proprioceptive signals, and as reflex muscle tension, spasm and finally, muscle pain occur [28, 36, 40, 51, 55]. Parafunctions of masticatory muscles are expressed by a great variability of symptoms: gritting of teeth, uncontrolled movement of the mandible forward or to the side; inter- 
position of the tongue between the teeth; biting the tongue and lips; squeaking the teeth; rhythmic movements of the tongue and sub maxillary muscles [21, 36, 42, 59, 60]. One of the varieties of masticatory muscles activity is bruxism subconscious, uncontrolled episodes of pressing and grinding teeth. Main causes of bruxism are psychological stress factors of the patient [55]. Stress often leads to an increased muscle fibre activity in chewing muscles [33, 37, $43,50,56]$.

The chewing muscles are controlled by the trigeminal nerve (motoric portion of mandibular nerve) and the trigeminal central nuclei are linked to the reticular system, which is responsible to transfer signals of alarm such as fear, anger, pain and increased of tonus. The so developed muscle power exceeds the required force for mastication. Dental abrasion, chipping, hyper sensibility and periodontal trauma are some possible consequences [2, 3, 6, 9, $12-16,26,27,48,55]$.

The key role of masticatory muscle hypertonus in development of TMJ pain dysfunction syndrome is reflected widely in the literature $[5,24,26,27,47,56,58]$. Disc dislocation and muscle symptoms are the most frequent diagnosed signs of craniomandibular disorder [33, 34].

TMJ dysfunction clinically might be accompanied by myofascial pain syndrome (MPS), conditioned by the local muscle hyper tones (myofascial trigger points) in masticatory muscles. MPS can be found in about $40 \%$ cases of prosopalgias (trigeminal neuralgia) $[17,18,20,23]$. Orthodontic changes in situations with increased muscle activity are capable to aggravate craniomandibular dysfunction and may complicate planned orthodontic manipulations [1, 4, 13, 33, 34, 46].

Treatment of hypertonic chewing muscles is aimed to relax muscles and eliminate muscle pain. There is still no uniform opinion on the optimal treatment regime. The proposed methods, including prosthetics, orthopedic, orthodontic, surgical and physiotherapeutic are controversially discussed in the literature. Interferences (5\%), post-filling tooth sensitivity and pain (3\%), psychogenic pain (3\%), post-herpetic prosopalgias (3\%) and senestopathy (hallucination of deep sensation) (3\%) are reported adverse events and side effects during and after treatments mentioned before $[12,25,26,41,46,53]$.

Botulinum Toxin Type A (BTA) has been emerged at an accelerated pace during the past 20 years. New therapeutic areas have been explored and neurological, cosmetic, urinary, gastrointestinal and pain-related conditions indications for BTA have been introduced $[2-4,10,20,35,36,38-41,44,51,52$, 56, 57].

Clostridium botulinum, a gram-positive anaerobic bacterium produces Botulinum toxin (BTX). BTX causes botulism with clinical symptoms after the intake of contaminated food or wound infection. BTX consists of 7 neurotoxins, which are indicated as types A, B, C, D, E, F and G. Type C has two subtypes, $\mathrm{C} 1$ and $\mathrm{C} 2$. The structure of the BTX types is similar, but distinction can be done by antigenic and serologic attributes. Human botulism is provoked primarily by types A, B and $\mathrm{E}$ (infrequently F), whilst Types $\mathrm{C} 1$ and $\mathrm{C} 2$ cause animal toxicities only. A light chain with approximately $50 \mathrm{kDa}$ and a heavy chain with $100 \mathrm{kDa}$ configure the entire molecule. The light chain acts as a zinc endopeptidase with proteolytic activity. The heavy chain is in charge for binding the toxin to the presynaptic receptors with cholinergic specificity (Fig. 1a, b).

The aim of this study was to evaluate the efficiency and duration of reduced or eliminated hypertonus of chewing muscles measured with surface Electromyography (EMG) in two groups. A randomized controlled clinical trial was performed, and the participants, after given their informed consent, were randomly assigned into either subgroup A (patients, who were treated with myo-relaxation medication and relaxing splint) or subgroup B (patients, who were treated with Botulinum Toxin A (BTA) as injection to affected muscles). a

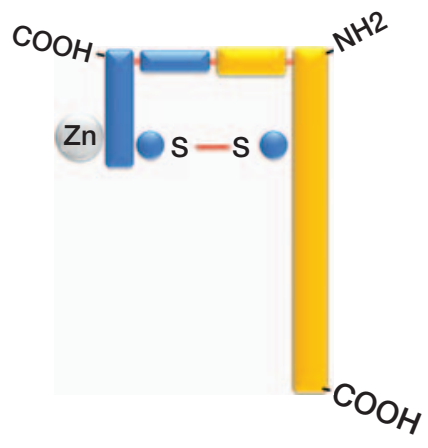

b

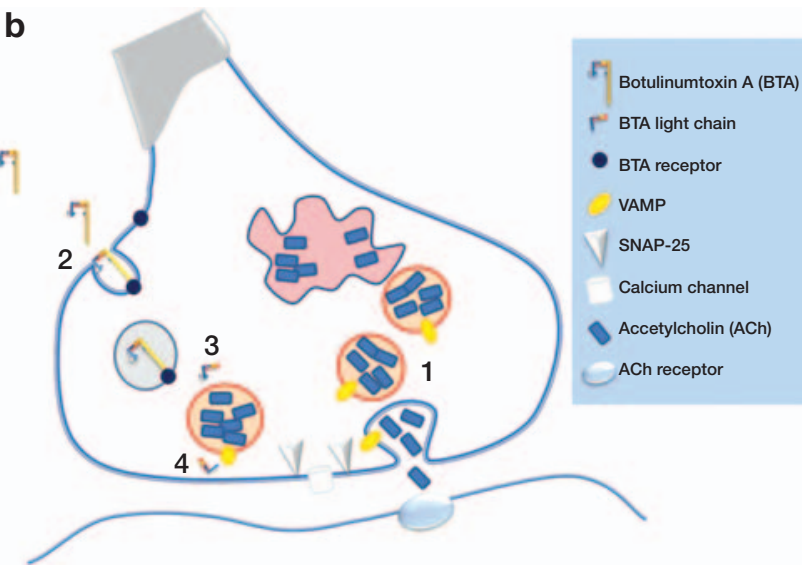

Fig. 1: (a) The structure of the Botulinumtoxin A (BTA) molecule. The BTA molecule consists of a light chain with approximately 50 kDa and a heavy chain with $100 \mathrm{kDa}$. The light chain acts as a zinc endopeptidase with proteolytic activity. The heavy chain is in charge for binding the toxin to the presynaptic receptors with cholinergic specificity. (b) Mechanism of action of Botulinumtoxin A (BTA) on the neuromuscular end plate: 1) The normal course of Acetylcholin (ACh) transport: vesicle associated membrane protein (VAMP) and synaptosomal-associated protein 25 (SNAP 25) are proteins and essential for membrane fusion. The synaptic vesicle, carrying ACh and the plasma membrane are fused under assistance of a firm compound of VAMP and SNAP 25. ACh is released into the synaptic gap. 2) BTA is incorporated after docking to the BTA receptor via endocytosis. 3) The light chain of the BTA molecule is separated and released into the zytosol. 4) The light chain interacts with the SNAP protein (proteolysis) and the fusion of the synaptic vesicle with the plasma membrane is prohibited 


\section{Material and method}

Patients diagnosed with severe bruxism and patients with local muscle dystonia were included in the study. Eligible patients were assigned either in the bruxism group (36 persons: 26 females, 10 men with the diagnosis "bruxism" and "pressing") or in the focal muscle dystonia group (31 persons: 21 females, 10 men) with diagnosis of focal muscle dystonia.

Electromyography was performed following the standard protocol. The following measurements were used to evaluate the treatment effect on EMG activity of the chewing muscles:

POC. The EMG potentials from the paired muscles are compared by calculating a percentage overlapping coefficient (POC, \%) [10]. POC is an index of the symmetric distribution of the muscular activity as determined by occlusion. The index ranges between $0 \%$ and $100 \%$ : when two paired muscles contract with perfect symmetry, POC is $100 \%$.

TORS. Torque coefficient (TORS, \%) is calculated by superimposing the right temporalis plus left masseter normalized EMG amplitudes over the left temporalis plus right masseter normalized EMG amplitudes. TORS ranges between 0\% (complete presence of lateral displacing force) and $100 \%$ (no lateral displacing force). TORS values are below $10 \%$ under normal circumstances.

CER.LOAD. Is assessed as the percentage ratio between the SCM muscle potentials recorded during maximum voluntary clenching and the muscle potentials obtained during the maximum contraction task. Normally, CER.LOAD value is below $20 \%$.

IMPACT. Shows the total biopotential of an examined muscle. Absolute values vary between $500 \mathrm{mV}$ and $2500 \mathrm{mV}$.

SIM. An indicator for masticatory symmetry. Frequency, amplitude, phase and total biopotential of a chewing sequence are taken into consideration.

Clinical features and baseline data of patients in the bruxism group: 10 patients (28\%) showed grinding teeth at nights; 8 patients (22\%) pressed during daytime in response to any light emotional stressor; 10 patients (28\%) complained of breakage of dental restorations. Episodic facial pain of muscle origin (myofascial painful syndrome of the face), headaches, conditioned by the tension of pericranial muscles were typical anamnesis data within these subjects. Eight patients (22\%) reported previous orthodontic treatment. All patients reported fatigue masticatory muscles in the mornings and hyper sensibility of teeth. Missing teeth have been found frequently, as well as pathological abrasion of teeth with the typical cervical lesions and gum recessions. A reduced vertical dimension with a diminished lower facial height in combination of hypertrophic masseter muscles could be examined frequently (Figs. 2, 3). Painful indurations (myofascial trigger points) within the masticatory muscle could be detected by palpation. Ten patients $(28 \%)$ reported pain irradiating to upper and low jaw, ear, front area, TMJ and throat. Palpation in the area of tuber maxillae was rather painful in 28 patients (77\%); pain and discomfort occurred in the area of medial alar muscle and digastrics muscle. All patients had tense muscles of the floor of the mouth and the hyoid bone showed restricted

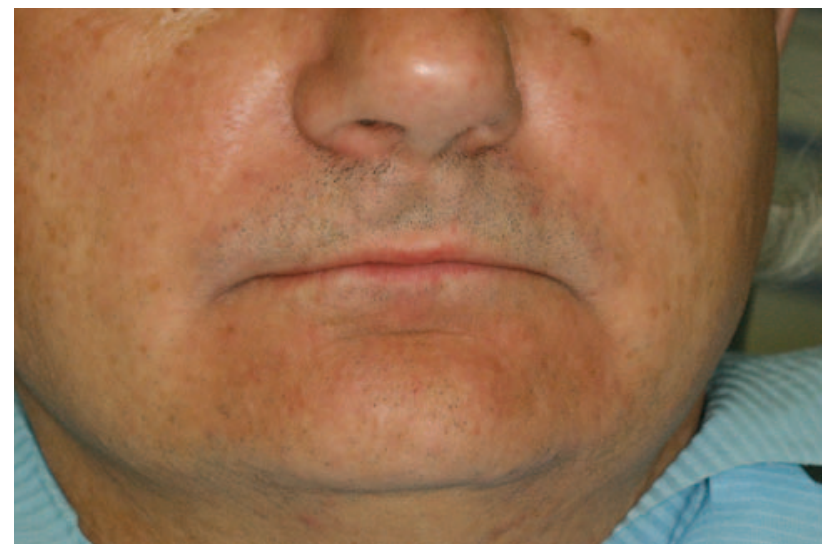

Fig 2: A typical clinical appearance of a patient with severe bruxism. Frontal view

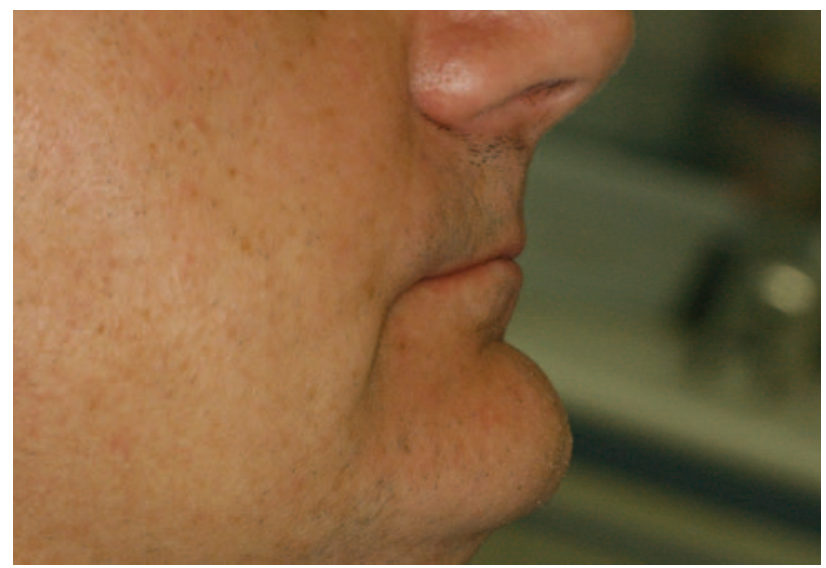

Fig 3: The same person as shown in Fig 2. Lateral view

mobility. Ten patients (28\%) presented with tension and a slight hypertrophy of the sternocleidomastoid muscle. A total of 14 patients (38\%) felt pain during palpation of the lateral pole of the joint.

Ten patients $(28 \%)$ had limited mouth opening due to pain. Further passive lowering of the mandible was practically impossible due to sharp, intensive pain. Nine patients (25\%) presented with clicking and crunching during mouth opening. Painful sensibility of the facial skin and changes of the oral mucosa have been diagnosed less frequently.

An asymmetric action of the temporal muscles (POC, 65-72\%), masseter muscles (POC, 58-70\%) and neck muscles (POC, 68-77\%) was identified with surface EMG. TORS was found to be $>15 \%$ and CER.LOAD $16-20 \%$. The total bio potential for the examined muscles was express by an IMPACT $3800-8200 \mathrm{mV}$. Masticatory symmetry was expressed by SIM $2-58 \%$. A total of 18 patients showed a unilateral mastication pattern.

Clinical feature and baseline data of the patients in the focal dystonia group: focal dystonia with the involvement of chewing and perioral muscles and clinical forms of oromandibular dystonia (OMD): spasm of muscles during mouth opening; pressing (dystonic trismus) with constant trismus and lateral spontaneous motions of the mandible (Fig. 4). All patients in 


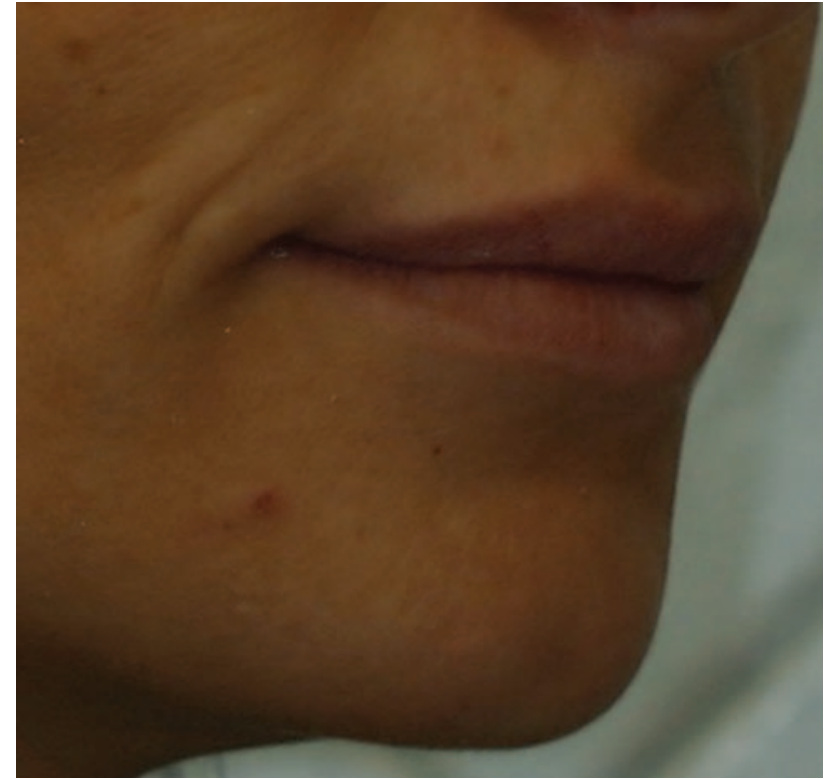

Fig 4: A typical clinical appearance of a patient with focal perioral dystonia. Frontal view

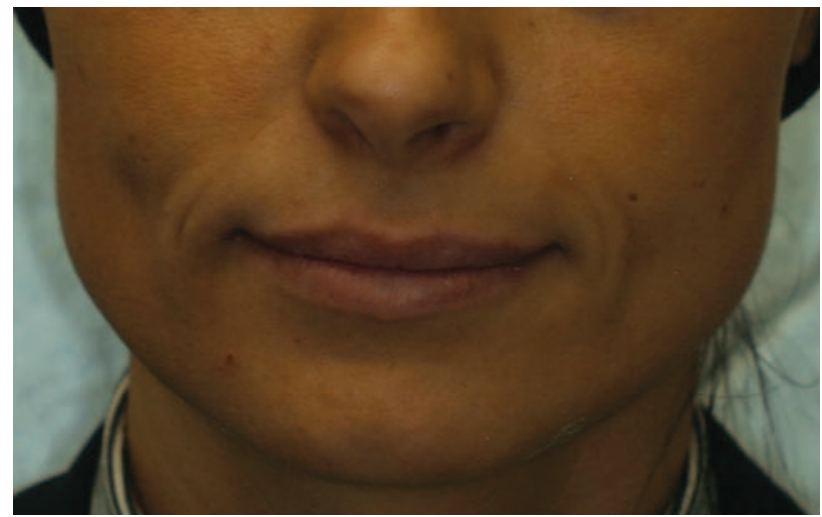

Fig 5: The same person as shown in Fig 4. The focal perioral dystonia in combination with a prominent masseter muscle is illustrated

this group reported unpleasant sensations, described as: "periodical motions of the mandible", "maxilla goes aside", "it is impossible to find a comfortable position of the mandibula", "forced squeezing the teeth" and "teeth chattering". Some patients within this group reported previous failed implantation. The average duration of pain was over 3 months. Signs of hypertrophy (masseter, temporalis muscle) have been detected clinically, in combination with density of the muscles and painful indurations (myofascial trigger points) (Fig. 5). Considerably changes were more often detected in the masseter muscle, fewer changes in temporal and pterygoid muscles. Five patients presented with an asymmetric character of dystonia, in combination with a visible asymmetry of the lower face (due to the different volume of the hypertrophied masseter muscle). A visible contraction of the platysma muscle could be correlated to pressing and grinding pattern of the individual. The mobility of the head was restricted in all patients. All participants within this group showed signs of dystonia in other areas of the body, such as blepharospasm; light and moderate forms of cervical dystonia; dystonic tremor

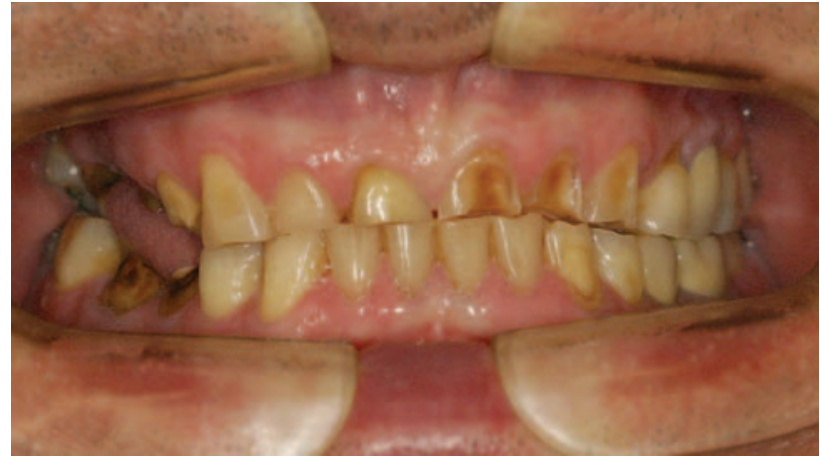

Fig 6: Intraoral view of a typical intraoral picture: Missing teeth, pathological abrasion and cuneiform defects in cervical region are characteristic in the focal dystonia group

of the head and upper extremities; writing cramp (Fig. 2). Missing teeth, pathological abrasion of teeth and presence of cuneiform defects in cervical area could be detected in all patients of this group (Fig 6).

Nine patients $(29 \%)$ had structural changes of temporomandibular joint in the form of chronic displacement of the disc and signs of osteoarthritis, confirmed by magnetic resonance imaging.

The following values had been recorded with surface EMG of masticatory muscles and neck muscles: POC temp. 65-72\%; POC mass. (58-70\%); POC scm (53-66\%); TORS $>15-25 \%$; CER.LOAD 23-98\% and IMPACT -3800-8200 mV; SIM (2-58\%). Most of the members of this group showed a single-sided mastication performance.

Intervention: To evaluate the efficiency and duration of reduced or eliminated hyper tonus of the masticatory muscles under control of the surface EMG in the 1st and 2nd group, the participants were randomly assigned to two different treatment groups: treatment group A (myo-relaxation medication and relaxation splint), treatment group B (injection of Botulinum Toxin A (BTA) to affected muscles).

The surface EMG of masseter, temporal and neck muscles was performed before and during the treatment (Days 1, 2, 3, 7, 14, 21 and 30). In addition a follow-up EMG examination was performed after 6 months. Injections were made directly to the affected muscles, bilaterally and symmetrically. A transcutaneous or intra oral approach was chosen for masseter muscle (dose 30-50 units BTA per side). The dose for temporal muscles was 5-20 units BTA per side. Target muscle areas for injections were chosen on clinical findings and EMG signals and in accordance to standard recommendations for the therapeutic use of Botulinum Toxin. Injections were made in the outpatient department after signing the informed consent form. There were no complications and adverse events in any case and no additional treatment or interventions were required after the use of BTA. The treatment in group A was performed in accordance to the current recommendations on myo-relaxation medication prescription and relaxation splint therapy.

\section{Results}

Patients in treatment group A (myo-relaxation medication and relaxation splint) showed only a short and limited (in terms of 


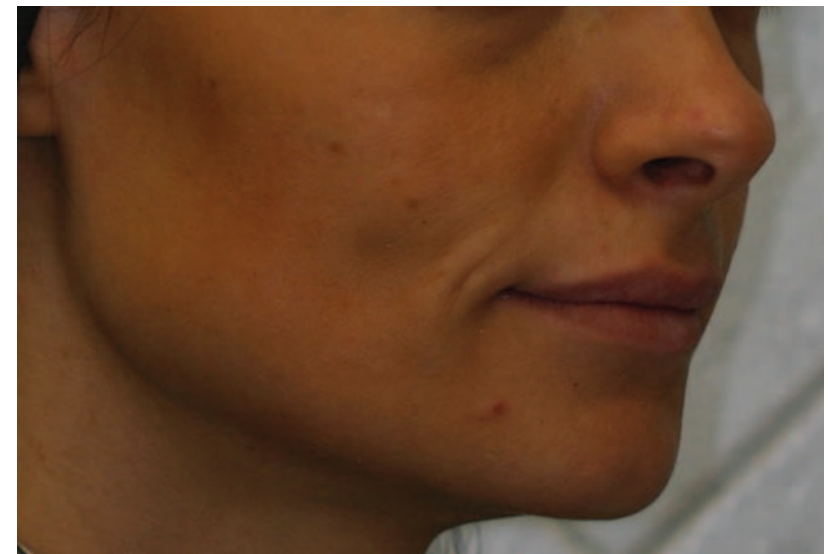

Fig 7: The same person as shown in Fig 5 after the treatment with Botulinum Toxin A

time) response in objective muscle relaxation. A short and limited reduction of the EMG activity was observed under therapy within 10-14 days, but after one month, the levels of EMG activity reached their initial values. Muscle relaxing effects were achieved, but they were minor and limited. A possible explanation for this finding is that the neuromuscular system adapts to the new occlusal situation on the splint, and develops new muscular driven habits, which serve as modulator for an increased muscle tonus [54].

Clinical evaluation of the patients in treatment group B (injection of Botulinum Toxin A to affected muscles) showed reduced intensity of pain, increased quantity of mandibular movements, increased mouth opening and improvement of chewing function on days 7 and 14 after injection, the maximal effect was achieved 21-30 days after injection. No relapse (facial dyskinesia) was seen within the short-term follow-up (6 months) (Fig 7).

The EMG changes in subgroup B, treated by BTA could be detected on the $3^{\text {rd }}$ day already. The total biopotential of the examined muscles reduced from the initial value of $8900 \mathrm{mV}$ to $5600 \mathrm{mV}$, still above the normal value of $2500 \mathrm{mV}$. A continuous reduction was observed within the next 14 days. Dynamic control within 6 months (1 time a month EMGexamination was made) showed that a myo-relaxing effect was preserved.

\section{Conclusion}

The study demonstrated the positive impact of BTA on EMG-characteristics, reflecting the functional condition of facial muscles. BTA injections seem to have a positive effect in patients with bruxism and local muscle dystonia. An increased quantity of mandibular movements and a better coordination can be observed after BTA injection. In comparison with a standard myo relaxation therapy, the effects of BTA treatment are more distinct and long standing. Within this study, the therapeutic effects of the allocated treatment regimes were measured with EMG surface electrodes.

A possible explanation for the observed effects is the discontinuation of the vicious circle stereotype mandibular movement - muscle spasm - muscle pain - amplified muscle spasm - altered mandibular movements and so on.

BTA appears to be a promising therapeutic regime in patients with focal muscle dystonia and oral dyskinesia. A confirmed diagnosis and lege artis application by experienced clinicians constitute as sine qua non. Further studies are required to improve our knowledge in Botulinum Toxin A treatment of muscle dystonia and dyskinesia.

\section{Conflict of interest}

The authors declare that there is no conflict of interest.

\section{References}

[1] Al Halef Naef. Orthodontic therapy of the patients with central disocclusion of dentitions in permanent occlusion using fixed equipment: Synopsis of a thesis of candidate of medical science Moscow, 2001.

[2] Acquardo MA, Borodic GE. Treatment of myofascial pain with botulinum A toxin. Anesthesiology 1994;80:705-6.

[3] Acquardo MA, Borodic GE. Basic and therapeutic aspects of botulinum and tetanus toxins. Intern. Conf., Orlando, USA Congress Summary. 1999; p. 91.

[4] Al-Ahmad HT, Al-Qudah MA. The treatment of masseter hypertrophy with botulinum toxin type A. Saudi Med J 2006;27: 397-400.

[5] Bragina OM. Study of early parodontium manifestations and tone of masticatory muscles of the patients with intact dentitions and the patients with partial loss of teeth. Nizhny Novgorod's Magazine of Medicine 2008;2:141.

[6] Budilina SM, Degtyareva VP. Physiology of maxillofacial area. Moscow Medicine 2001;87:156.

[7] Clark CL, Baur DA. Management of mentalis muscle dysfunction after advancement genioplasty: a case report. J Oral Maxillofac Surg 2004;62:611-3.

[8] Celic R, Jerolimov V, Panduric J. A study of the influence of occlusal factors and parafunctional habits on the prevalence of signs and symptoms of TMD. Int J Prosthodont 2002;15(1):43.

[9] Dworkin SF, LeResche L. Research diagnostic criteria for temporomandibular disorders. J Craniomand Disorders 1992;6: 301-55.

[10] Dressler D. Botulinum toxin therapy. N.Y.: Thieme; 2000.

[11] Devlikamova FI. Synopsis of a thesis for a doctor of medical science's degree Kazan, Russia; 2004.

[12] Forssel H, et al. Occlusal treatment in temporomandibular disorders: a qualitative systematic review of randomized controlled trials. Pain 1999;83:549-60.

[13] Glaros AG, et al. Effect of parafunctional clenching on temporomandibular disorder pain and proprioceptive awareness. Cranio 2000;18(3):198-204.

[14] Gurney CE. Chronic bilateral benign hypertrophy of the masseter muscle. Am Surg 1947;73:137.

[15] Gvozdeva JV. Comparative analysis of jawbone density and regional circulation's state of the young patients in the process of orthodontic therapy of dental anomaly: synopsis of a thesis of candidate of medical science Perm, Russia, 2003.

[16] Gribova NP, et al. Musculation of proper masticatory muscles of the patients with syndrome of pain-dysfunction of temporomandibular joint. Nizhny Novgorod's Magazine of Medicine 2008;2:216.

[17] Guseva IE. Assessment of dynamics of functional hyperemia in parodontium candidate of medical science's thesis, Moscow. 1991; p. 180.

[18] Ivanichev GA. Painful muscle induration. Kazan University Press; 1990. pp. 18-24.

[19] Ivanov VS. Periodontal diseases. Moscow: Moscow Medicine; 1989. p. 272.

[20] Jankovic J, Hallet M. Therapy with botulinum toxin. NY: Marcel Dekker; 1994. p. 525.

[21] Hanashima MK, et al. A study regarding occlusal plane and posterior disocclusion. IJSOM 2008;1(1):27-33. 
[22] Kaljuzhniy DV. Physiologic regulating mechanisms of algesthesia. Moscow: Moscow Medicine; 1989. p. 272.

[23] Karlov VA. Face Neurology. Moscow: Moscow Medicine; 1991.

24] Krechina EK. Modifications of clinical-functional state of teenagers' periodontium and their correction with the help of hypoxic stimulation Candidate of medical science's thesis, Moscow; 1998. p. 207.

[25] Khvatova VA. Clinical gnathology. Moscow: Moscow Medicine; 2005.

[26] Kuttila M, et al. TMD treatment need in relation to age, gender, stress and diagnostic subgroup. J Orofacial Pain 1998;12:67-74.

[27] Loginova NK. Functional presuppositions for elaboration of mechanic theory of etiology and periodontal diseases' pathogenesis. New Stomatol 1993;1:2-7.

[28] Loginova NK, Volozhin AI. Pathophysiology of periodontium (theory and practice). Study guide, Moscow, second edition; 1994. p. 108.

[29] Loginova NK. Functional diagnostics in Stomatology. Moscow: Partner 1994;77(4):4-6.

[30] Le KD, et al. Prevalence of primary focal and segmental dystonia in Oslo. Neurology 2003;61(9):1294-6.

[31] Melnichuk TA. Clinical-functional state of periodontal tissue during integrated therapy of periodontal diseases at pubertal and ephebic age Candidate of medical science's thesis. Kiev 1986.

[32] Mingazova LR. Pathogenesis and therapy of face myofascial painful syndrome. Materials of Sechenov's FPPOMMA Academy young scientists' clinical conference Moscow; 2002. pp. 54-8.

[33] Mingazova LR. Clinical-physiological analysis and therapy of face myofascial painful syndrome Candidate of medical science's thesis. Moscow, 2005.

[34] McGregor NR, et al. Pain intensity, illness duration, and protein catabolism in temporomandibular disorder patients with chronic muscle pain. J Orofac Pain 2003;17(2):117-24.

[35] Moore AP, Wood GD. The medical management of masseteric hypertrophy with botulinum toxin type A. Br J Oral Maxillofac Surg 1994;32:26-8.

[36] Mantell AM. Dilution, storage, and electromyographic guidance in the use of botulinum toxins. Dermatol Clin 2004;22:135-6.

[37] Miller JR, Mancl L, Critchlow C. Severe retrognathia as a risk factor for recent onset painful TMJ disorders among adult females. J Orthod 2005;32(4):249-56.

[38] Nixdorf DR, Heo G, Major PW. Randomized controlled trial of botulinum toxin A for chronic myogenous orofacial pain. Pain 2002;99:465-73.

[39] Orlova OR. Focal dystonia: clinic, pathogenesis, therapy using botulinum toxin Dissertation for a doctor of medical science's degree, Moscow, 2000.

[40] Orlova OR, Mingazova LR, Vein AM. Facial pain of muscle type: clinical-physiological peculiarities and therapy using botulinum toxin type A. Russian research-to-practice conference "Clinical and theoretic aspects of acute and chronic pain". Nizhny Novgorod 2003;113-5.

[41] Orlova OR, Yakhno NN. Usage of botox (botulinum toxin type A) in clinical practice, Moscow, 2001.
[42] Orlova OR, Mingazova LR, Vein AM. Face myofascial painful syndrome: new aspects of clinic, pathogenesis and therapy. New Stomatol 2003;25-9.

[43] Ohshima S, et al. Prolonged effects of hypofunction on the mechanical strength of the periodontal ligament in rat mandibular molars. Arch Oral Biol 1991;36(12):905-11

[44] Pidcock FS, Wise JM, Christensen JR. Treatment of severe posttraumatic bruxism with botulinum toxin-A: a case report. J Oral Maxillofac Surg 2002;60:115-7.

[45] Petrov EA. Electrophysiological characteristics of painful syndrome temporo-mandibular joint's dysfunction. Russian Stomatology Magazine 2002;6:34-5.

[46] Petrosov YA, Skorikova LA. Prevention of dysfunction of temporomandibular joint by removal of masticatory muscles' parafunctions All-Russian Congress of Dentists: Prevention of stomatology diseases. Novosibirsk - Moscow 1988;156-7.

[47] Puzin MN. Neurostomatology diseases. Medicine 1997;12-48.

[48] Puzin MN, Vyazmin A. Painful dysfunction of temporo-mandibular joint. Moscow; Medicine, 2002;160.

[49] Uhac I, Kovac Z, Valentic-Peruzovic M, Juretic M, Moro LJ, Grzic R. The influence of war stress on the prevalence of signs and symptoms of temporomandibular disorders. J Oral Rehabil 2003;30:211-7.

[50] Ramfjord SP, Ash MM. Occlusion Philadelphia, Saunders Co. 1966

[51] Scott AB. Botulinum toxin injection into extraocular muscles as an alternative to strabismus surgery. J Pediatr Ophthalmol Strabismus 1980;17:21-5.

[52] Schwartz M. Treatment of temporomandibular disorders with botulinum toxin. Clin J Pain 2002;18(6):198-203.

[53] Suvinen TI, et al. Temporomandibular disorders. Part I: A comparison of symptom profiles in Australian and Finnish patients. J Orofacial Pain 1997;11:58-66.

[54] Slavicek G. The use of intraoral splints: a case report. IJSOM 2009; 2(2):73-81.

[55] Slavicek R. The masticatory organ: Function and Dysfunction. Gamma Med.-wiss. FortbildungsgmbH, Klosterneuburg, 2006.

[56] Tsaqi CY, et al. Effects on craniofacial growth and development of unilateral botulinum neurotoxin in injection into the masseter muscle. Am J OrthodDentofacial Orthop 2009; 135(2):142-6.

[57] Tsai CM, et al. Human masticatory muscle activity and jaw position under experimental stress. J Oral Rehabil 2002;29(1):44-51.

[58] Von Lindern JJ, et al. Type A botulinum toxin for the treatment of hypertrophy of the masseter and temporal muscles: an alternative treatment. Plast Reconstr Surg 2001;107:327-32.

[59] Winocur E, et al. Oral motor parafunctions among heavy drug addicts and their effects on signs and symptoms of temporomandibular disorders. J Orofac Pain 2001;15(1):56-63

[60] Winter-Defazio G, et al. Epidemiology of primary dystonia. Lancet Neurol 2004;3(11):673-8.

[61] Weisskircher HW. Myofasziale schmerzen und die Behandlung der Triggerpunkte. Zahnärztliche Mitteilungen 1999;8:36-40. 